\title{
CREBBP knockdown suppressed proliferation and promoted chemo-sensitivity via PERK-mediated unfolded protein response in ovarian cancer
}

\author{
Haoyang $\mathrm{Hu}^{1 *}$, Sheng Yin²*, Ruyue Ma1 ${ }^{2}$, Rujun Chen ${ }^{1}$, Shuqing $\mathrm{Li}^{3}$, Yaping Chen ${ }^{1}$, He Fei ${ }^{1 凶}$ and Lina Yang ${ }^{\circledR}$ \\ 1. Department of Obstetrics and Gynecology, Shanghai Fifth People's Hospital, Fudan University, 801 Heqing Road, Shanghai, People's Republic of China. \\ 2. Department of Obstetrics and Gynecology, Zhongshan Hospital Fudan University, 180 Fenglin Road, Shanghai, People's Republic of China. \\ 3. Obstetrics and Gynecology Hospital of Fudan University, 128 Shenyang Road, Shanghai, People's Republic of China. \\ *These authors contributed equally to this work.
}

$\square$ Corresponding authors: Lina Yang, Department of Obstetrics and Gynecology, Shanghai Fifth People's Hospital, Fudan University, 801 Heqing Road, Shanghai, 200240, People's Republic of China, Phone: +86 213490 4800, E-mail: linayang12@fudan.edu.cn. He Fei, Department of Obstetrics and Gynecology, Shanghai Fifth People's Hospital, Fudan University, 801 Heqing Road, Shanghai, 200240, People's Republic of China, Phone: +86 2124289559 , E-mail:18111250005@fudan.edu.cn.

(c) The author(s). This is an open access article distributed under the terms of the Creative Commons Attribution License (https://creativecommons.org/licenses/by/4.0/). See http://ivyspring.com/terms for full terms and conditions.

Received: 2020.11.19; Accepted: 2021.05.19; Published: 2021.06.01

\begin{abstract}
CREBBP, in short CBP, has been reported to be involved in tumorigenesis in various cancers, but its role in ovarian cancer remains largely unexplored. In our study, survival analysis of CBP in patients with ovarian cancer was conducted using the Kaplan-Meier Plotter database, then we utilized specific shRNA targeting CREBBP to block the expression of CBP, and detected its effect on cell proliferation and chemo-sensitivity in ovarian cancer cells. The results showed that high expression of CBP was correlated with poor prognosis in ovarian cancer patients. CREBBP knockdown in ovarian cancer cells significantly inhibited tumor proliferation both in vitro and in vivo. Moreover, CREBBP knockdown promoted chemo-sensitivity in ovarian cancer cells. Mechanism research further demonstrated that CREBBP knockdown attenuated unfolded protein response (UPR), which was mediated by PERK/ATF4/STC2 signaling pathway. Our research linked CBP and UPR in ovarian cancer and may provide new strategies for the clinical treatment of ovarian cancer.
\end{abstract}

Key words: CREBBP; UPR; chemo-sensitivity; apoptosis; ovarian cancer

\section{Introduction}

Ovarian cancer is one of the five leading causes of cancer deaths among American women [1], and also the $8^{\text {th }}$ leading cause of death worldwide in 2020 [2]. Epithelial ovarian cancer (EOC) accounts for over $95 \%$ of ovarian malignancies, and high grade serous ovarian cancer is the most common histologic subtype, accounting for over $70 \%$ of EOC [3]. EOC generally presents at an advanced stage with the 5 -year overall survival rate from $30 \%$ to $40 \%$ [4]. It is urgent to develop new strategies for early diagnosis and treatment of ovarian cancer.

The CREB-binding protein (CREBBP, in short $\mathrm{CBP})$ is a transcriptional co-activator with lysine acetyltransferase activity. CBP has been reported to be involved in various cellular processes including cell metabolism, embryonic development and cell differentiation [5-8]. Previous research on the role of CBP in tumorigenesis were mainly derived from gene mutation or chromosome arrangement [9-11]. Recently, studies to investigate the function of CBP on epigenetic modification or directly acetylation have attracted more and more attention. CBP and PTEN knockdown alone or together could increase the H3K27me3 levels and inhibit H3K27Ac in prostate cancer [12]. Crebbp knockdown in small cell lung cancer cells reduced H3K27Ac levels leading to epigenetic suppression of $\mathrm{CDH1}$ and other cell adhesion-associated genes [13]. CBP directly acetylated BCAT2 to promote protein degradation [14], CBP dramatically enhanced DOT1L acetylation and promoted protein stability [15] and CBP directly acetylated KDM2B and promoted target gene 
transcription [16]. The aforementioned study demonstrated that CBP could serve as a tumor suppressor or an oncogene in different cancer types. Till now, the role of CBP in ovarian cancer, especially in epithelial ovarian cancer has not yet been fully elucidated.

Endoplasmic reticulum (ER) is an organelle for protein folding. Once misfolded proteins or unfolded proteins accumulated, the unfolded protein response (UPR) is triggered to maintain ER homeostasis and protect cell from death. Generally, The UPR is mediated by three ER transmembrane sensors: PERK, IRE1a and ATF6a [17]. PERK activates eIF2a phosphorylation to attenuate global protein translation but selectively enhances ATF4 and subsequently downstream effectors such as STC2 [18]; IRE1a mediates cleavage of X-box binding protein ( $X X B P 1)$ mRNA into spliced XBP1 (sXBP1) mRNA, which is translated into a potent transcriptional activator that induces the expression of UPR responsive genes; ATF6a dissociates from GRP78 and translocates to the Golgi where ATF6a is cleaved and activated, the activated ATF6a translocates to the nucleus and activates the transcription of target genes. The three signaling pathways are intertwined to promote cell survival [19-21].

In the present study, we utilized shRNA targeting $C R E B B P$ to inhibit CBP expression and examined its effect on ovarian cancer cells. The results showed that CREBBP knockdown suppressed tumor proliferation and promoted chemo-sensitivity via mitigating the PERK-mediated unfolded protein response (UPR). Our results may provide new cues for the clinical treatment of ovarian cancer.

\section{Materials and methods}

\section{Reagents and antibodies}

RPMI-1640 cell culture medium and DMEM cell culture medium were purchased from Sigma-Aldrich Inc. (St. Louis, MO, USA). Fetal bovine serum (FBS) and Plasmocin ${ }^{\mathrm{TM}}$ were purchased from Thermo Fisher Scientific (Waltham, MA, USA). LipoFiter ${ }^{\mathrm{TM}}$ Liposomal Transfection Reagent was purchased from Hanbio Biotechnology (Shanghai, China). CDDP was purchased from Selleck Chemicals (Houston, TX, USA). Antibodies used were listed in Table 1. HRP-conjugated goat anti-rabbit and anti-mouse secondary antibodies were from Sigma-Aldrich Inc. (St. Louis, MO, USA). EnVision ${ }^{\mathrm{TM}}$ III Detection System (GK500705) was purchased form Gene Tech (Shanghai, China).

\section{Cell lines and cell culture}

Human ovarian cancer cell lines (HEY and SKOV3) and human embryonic kidney 293T cell line were obtained from ATCC. Ovarian cancer cells were cultured in RPMI-1640 medium supplemented with $10 \%$ FBS. 293T cells were grown in DMEM medium with $10 \%$ FBS. All the cells were cultured at $37^{\circ} \mathrm{C}$ in a $5 \% \mathrm{CO}_{2}$ humidified incubator. STR DNA profiling was used for cell line authentication. Plasmocin ${ }^{\mathrm{TM}}$ was used for removal of mycoplasma in cell culture.

\section{Construction of CREBBP knockdown plasmid}

The pLKO.1 - TRC cloning vector was obtained from Addgene (Cambridge, MA, USA). shRNA target sequences are as follows: shCBP-1: GCGGAGCCATC TAGTGCATA; ShCBP-2: GCAAGACATCCCGAGTC TATA; shLuc (Ctrl): CTTACGCTGAGTACTTCGA. The experiment was conducted according to the Addgene's protocol. The positive clones were verified by sequencing.

\section{Establishment of CREBBP knockdown cells}

The pLKO.1 construct was co-transfected into 293T cells with psPAX2 and pMD2.G by LipoFiter ${ }^{\mathrm{TM}}$ (Hanbio, Shanghai, China). The supernatants containing virus particles were harvested after transfection for $48 \mathrm{~h}$, and filtered through a $0.45 \mu \mathrm{m}$ filter to remove the cell debris. Then the virus particles were used to infect cancer cells along with polybrene $(8 \mu \mathrm{g} / \mathrm{mL})$. Subsequently, stable cell lines were selected with $1 \mu \mathrm{g} / \mathrm{mL}$ puromycin for 2 weeks.

\section{qRT-PCR}

Total RNA was extracted using TRIzol reagent (Thermo Fisher Scientific, Waltham, MA, USA). Then $1 \mu \mathrm{g}$ of total RNA was reverse transcribed into cDNA using the PrimeScript RT Reagent Kit with gDNA Eraser (Takara, Dalian, China). qRT-PCR was performed using the FastStart Universal SYBR Green Master (Roche Diagnostics, Indianapolis, IN, USA) in the Applied Biosystems 7500 Real-Time PCR System. The primer sets used were listed as follows: sense primer for CREBBP, 5'-CGGCTCTAGTATCAACCC AGG-3', and antisense primer for CREBBP, 5'-TTTTG TGCTTGCGGATTCAGT-3'; sense primer for GAPDH, 5'-TTGAGGTCAATGAAGGGGTC-3' and antisense primer for GAPDH, 5'-GAAGGTGAAGGTC GGAGT A-3'. Relative mRNA levels were analyzed using the Comparative $\mathrm{Ct}$ Threshold Method $(\triangle \Delta \mathrm{CT})$ with GAPDH as a reference gene.

\section{CCK-8 assay for cell proliferation}

The indicated cells were seeded at a density of 700 cells per well in 96-well plates. Then the cells were incubated with $10 \mu \mathrm{L}$ CCK- 8 per well for $2 \mathrm{~h}$ at the same time on day 1,2,3,4 and 5. The plates were then shaken for $10 \mathrm{~min}$, and the optical density (OD) at 450 $\mathrm{nm}$ was measured using a microplate reader. Each sample was analyzed three times in quintuplicate. 
Table 1. Antibodies lists

\begin{tabular}{llll}
\hline Antibodies & Work Dilution & Catalog & Source \\
\hline CBP & $1: 1000$ for WB; 1:100 for IHC & 7389 & Cell Signaling Technology, Danvers, MA, USA \\
GRP78 & $1: 1000$ for WB & sc-13968 & Santa Cruz Biotechnology, Dallas, TX, USA \\
ATF6a & $1: 1000$ for WB & sc-166659 & Santa Cruz Biotechnology, Dallas, TX, USA \\
$\beta$-actin & $1: 10000$ for WB & MABT825 & Sigma-Aldrich, St. Louis, MO, USA \\
STC2 & $1: 1000$ for WB & HPA045372 & Sigma-Aldrich, St. Louis, MO, USA \\
IRE1a & $1: 1000$ for WB & 3294 & Cell Signaling Technology, Danvers, MA, USA \\
PERK & $1: 1000$ for WB & 5683 & Cell Signaling Technology, Danvers, MA, USA \\
p-eIF2a & $1: 1000$ for WB & Cell Signaling Technology, Danvers, MA, USA \\
eIF2a & $1: 1000$ for WB & 5398 & Cell Signaling Technology, Danvers, MA, USA \\
ATF4 & $1: 1000$ for WB & 11815 & Cell Signaling Technology, Danvers, MA, USA \\
Cleaved Caspase 3 & $1: 1000$ for WB & Cell Signaling Technology, Danvers, MA, USA \\
PCNA & $1: 1000$ for IHC & Abcam, Cambridge, MA, USA \\
\hline
\end{tabular}

\section{Colony formation assay}

Cells were plated in 6-well plates at a density of 500 cells per well and allowed to grow for 10 days to form obvious clones. Then the cells were stained with $0.1 \%$ crystal violet in methanol. After washed with double distilled water, the clones were imaged, and the numbers of clones were quantified and analyzed using Graphpad Prism version 8.0.2 (GraphPad Software Inc., San Diego, CA, USA). Each experiment was repeated three times.

\section{IC50 measurement}

The indicated cells were seeded at a density of 6000 cells per well in 96-well plates and incubated overnight. CDDP was added at a concentration of 0,1 , $2,4,8,16$ and $32 \mu \mathrm{M}$ respectively. After $48 \mathrm{~h}$, the cells were incubated with fresh medium containing $10 \mu \mathrm{L}$ CCK-8 per well for $2 \mathrm{~h}$. The plates were then shaken for $10 \mathrm{~min}$, and the optical density (OD) at $450 \mathrm{~nm}$ was measured using a microplate reader. Each sample was analyzed three times in quadruplicate.

\section{Examination of apoptosis}

Cells were plated in $6 \mathrm{~cm}$ dishes and incubated overnight. Then CDDP was added into the medium. After incubation for $48 \mathrm{~h}$, cells were collected by trypsinization and stained by the Annexin V-FITC/PI Apoptosis Detection Kit (YEASEN Biotechnology, Shanghai, China) according to the manufacturer's instruction. The samples were examined by using the Amnis FlowSight ${ }^{\circledR}$ Imaging Flow Cytometer, and analyzed by the IDEAS software. The experiment was repeated three times.

\section{Western blot assay}

Whole cell lysates were generated using RIPA lysis buffer (Beyotime Biotechnology, Jiangsu, China) and quantified by the BCA Protein Assay Kit (Beyotime Biotechnology, Jiangsu, China). Protein samples were separated in SDS-PAGE and transferred onto PVDF membrane. Then the membrane was blocked with $5 \%$ non-fat milk for $1 \mathrm{~h}$ at room temperature, and incubated with the indicated primary antibodies at $4{ }^{\circ} \mathrm{C}$ overnight with gently shaking. After washing in TBST, the membrane was incubated with HPR-conjugated secondary antibody for $1 \mathrm{~h}$ at room temperature. The protein bands were visualized using the enhanced chemiluminescence substrate kit (Millipore, Schwalbach, Germany) in the FluorChem E system.

\section{XBPI Splicing Assay}

RNA extraction and cDNA synthesis were performed as described in qRT-PCR. XBP1 cDNA was amplified by PCR with the PrimeSTAR GXL DNA Polymerase (Takara, Dalian, China). The primer set encompassing the spliced sequences of human XBP1 was as follows: sense primer, 5'-AAACAGAGTAGCA GCTCAGACTGC-3'; antisense primer, 5'-TCCTTCTG GGTAGACCTCTGGGAG-3'. The amplification products were further digested by PstI at $37^{\circ} \mathrm{C}$ for $1 \mathrm{~h}$, and subsequently separated by DNA electrophoresis on a $2 \%$ agarose gel containing Gelred (Biotium Inc, Heyward, California, USA). The primer set for GAPDH amplification was described in qRT-PCR.

\section{Xenograft model in nude mice}

The xenograft models were generated in female athymic nude mice. The assay was carried out with the institutional guidelines and approved by ECNU Multifunctional Platform for Innovation (011). Cells were harvested by trypsinization and resuspended in $1 \times$ PBS, then subcutaneously injected into 6-week-old $\mathrm{BALB} / \mathrm{c}$ athymic nude mice. Six mice were used per cell lines, each mouse received twice injection in bilateral flank and each injection contained $5 \times 10^{6}$ cells. Tumor growth was monitored until the end point, and tumor size was measured every 3 days using an electronic caliper. At the termination of the study (the $27^{\text {th }}$ day), the mice were euthanized with $\mathrm{CO}_{2}$ exposure and necropsied to collect the subcutaneous tumors. Tumor volume was calculated with the formula: $\mathrm{V}=\mathrm{L} \times \mathrm{W} \times \mathrm{H} \times 0.52$. 


\section{Immunohistochemistry}

The subcutaneous xenograft tumors were dissected and fixed in formalin. After dehydration and paraffin-embedded, the tumors were sliced into 5 $\mu \mathrm{m}$ thickness sections, and the sections were deparaffinized with xylene, rehydrated in graded alcohol solutions. One set of slides was stained with hematoxylin and eosin. Other two sets of slides were stained with antibodies against CBP and PCDA respectively according to the manufacturer's instruction. Briefly, endogenous peroxidase activity was quenched using 3\% $\mathrm{H}_{2} \mathrm{O}_{2}$ in methanol for 15 minutes at room temperature, antigen retrieval was performed by boiling the slides in $0.01 \mathrm{M}$ citrate buffer $(\mathrm{pH}$ 6.0). After blocking in 3\% BSA for $1 \mathrm{~h}$ at room temperature, the slides were incubated with antibodies against CBP (1:100) and PCNA (1:1000) respectively at $4{ }^{\circ} \mathrm{C}$ overnight and incubated with secondary antibodies conjugated HRP for $1 \mathrm{~h}$ at room temperature. All slides were developed with $\mathrm{DAB}$ chromogen supplied by the EnVision ${ }^{\mathrm{TM}}$ III Detection System and counterstained with hematoxylin. After dehydration in graded alcohol solutions and transparence in xylene, the slides were mounted with neutral gum and analyzed under a brightfield microscope.

\section{Statistical analysis}

Data were presented as mean \pm standard deviation (SD). Data between two groups were analyzed by student's $t$ test (two-tailed distribution). Data between three groups were analyzed by one-way ANOVA, followed by Dunnett's multiple comparisons test. Statistical significance is described as follows: ${ }^{*} \mathrm{P}<0.05$, ${ }^{* *} \mathrm{P}<0.01$ and ${ }^{* *} \mathrm{P}<0.001$. $\mathrm{P}<$ 0.05 was considered statistically significant. Statistical analysis and graph production were performed using Graphpad Prism version 8.0.2 (GraphPad Software Inc., San Diego, CA, USA).

\section{Results}

\section{High expression of CBP is correlated with poor prognosis in ovarian cancer patients}

To explore the prognostic value of CBP in patients with ovarian cancer, we analyzed the prognosis data in the Kaplan-Meier plotter database (http:// kmplot.com/analysis/). As shown in Fig. 1A, high expression of CBP was associated with poor PFS (Fig. $1 \mathrm{~A}, \mathrm{p}=0.00079, \mathrm{HR}=1.25$ ). Furthermore, high expression of CBP also predicted poor OS in ovarian cancer patients (Fig. 1B, $p=0.014, H R=1.19$ ). Taken together, these data suggested that CBP could serve as a promising prognostic marker in ovarian cancer.
A

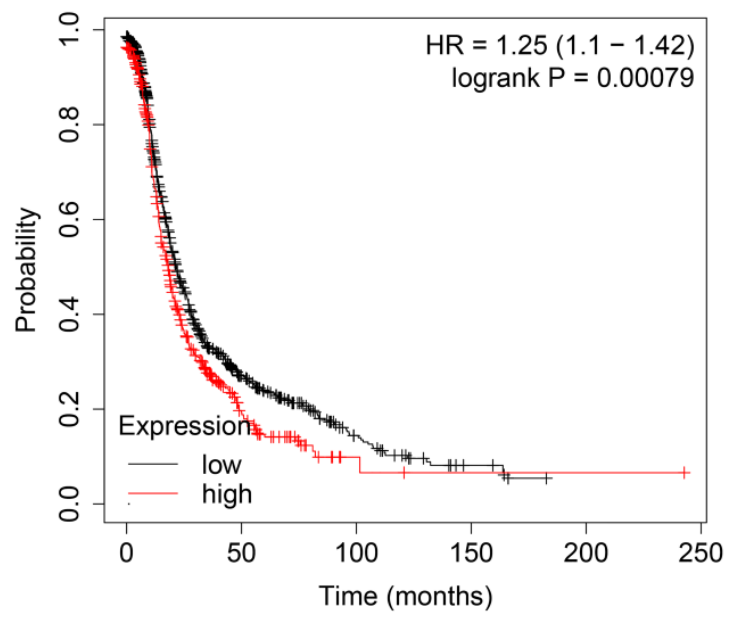

B

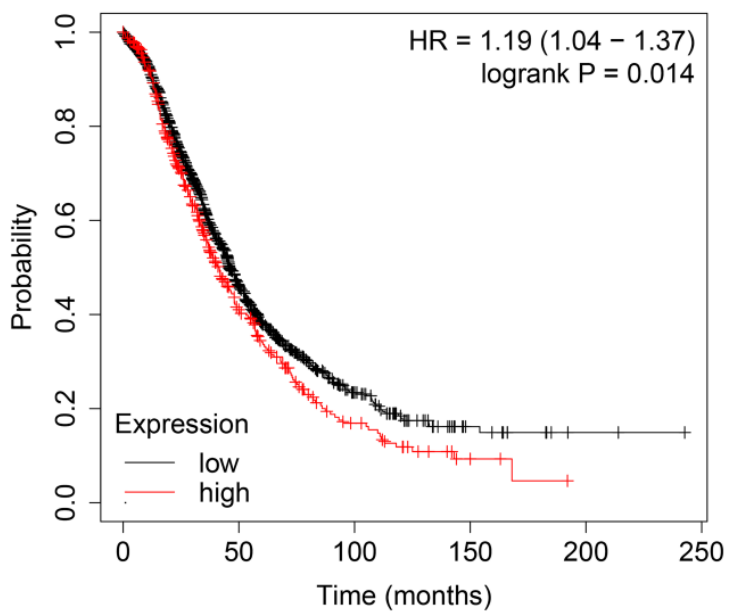

Figure 1. High expression of CBP predicts unfavorable prognosis in patients with ovarian cancer. A. High expression of CBP predicts poor PFS in patients with ovarian cancer. B. High expression of CBP predicts unfavorable OS in patients with ovarian cancer. PFS, progression free survival; OS, overall survival; HR, hazard ratio.

\section{CREBBP knockdown inhibits cell proliferation in ovarian cancer cells}

To investigate the role of CBP in ovarian cancer, two common ovarian cancer cell lines (HEY and SKOV3) were selected and infected with lentivirus harboring CBP shRNA (shCBP-1 and shCBP-2, respectively), and the control cells were infected with shRNA targeting firefly luciferase (shLuc). To detect the knockdown efficiency of shRNA, we collected whole cell lysates for western blot and total RNA for qRT-PCR. As the results shown, the two shRNAs significantly reduced CBP protein level (Fig. 2A) and decreased the level of CREBBP mRNA (Fig. 2B). Since $\mathrm{CBP}$ is a major transcription co-activator that regulates tumorigenesis, we examined the effect of CBP on proliferation in ovarian cancer cells using CCK-8 assay. The result indicated that CREBBP knockdown suppressed tumor proliferation both in HEY and SKOV3 cells (Fig. 2C and 2D). The colony 
formation assay was utilized to examine the clonogenic potential of cells, and the results also showed that CREBBP knockdown inhibited colony formation both in HEY and SKOV3 cells (Fig. 2E and 2F). Taken together, CREBBP knockdown significantly suppressed tumor proliferation in ovarian cancer cells.

\section{CREBBP knockdown inhibits tumorigenesis in xenograft tumor model}

To investigate the effect of $C R E B B P$ knockdown on tumorigenesis in vivo, a cell line-derived xenograft tumor model was established by subcutaneously injecting $C R E B B P$ knockdown and the corresponding control cells (HEY shCBP-1, HEY shCBP-2, and HEY shLuc) into the bilateral flanks of BALB/c nude mice. As shown in Figure 3A, knockdown of CREBBP by shCBP-1 completely suppressed tumor formation in all mice, and CREBBP knockdown by shCBP-2 significantly inhibited tumor growth. Both tumor volumes and tumor weights were dramatically reduced in CREBBP knockdown group (Fig. 3B and $3 C)$. Immunohistochemical staining for CBP and proliferation marker PCNA were further performed. As shown in Figure 3D, CREBB knockdown cells showed a reduced level of PCNA. Taken together, these results indicated that knockdown of CREBBP suppressed tumor growth in vivo.

\section{CREBBP knockdown promotes chemo- sensitivity and induces apoptosis in ovarian cancer cells}

To investigate the effect of CBP on chemosensitivity in ovarian cancer cells, we detected the viabilities of cells treated with CDDP at different concentrations. As shown in Figure 4A and Figure 4C, $C R E B B P$ knockdown enhanced the chemo-sensitivity of cells to CDDP both in HEY and SKOV3 cells (Fig. $4 \mathrm{~A}$ and $4 \mathrm{C}$ ). IC50 values were dramatically decreased in CREBBP knockdown cells (Fig. 4B and 4D). As
A



C

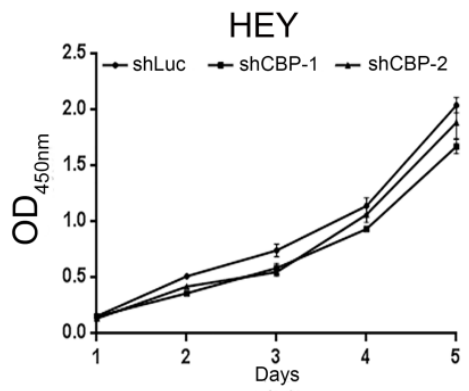

E

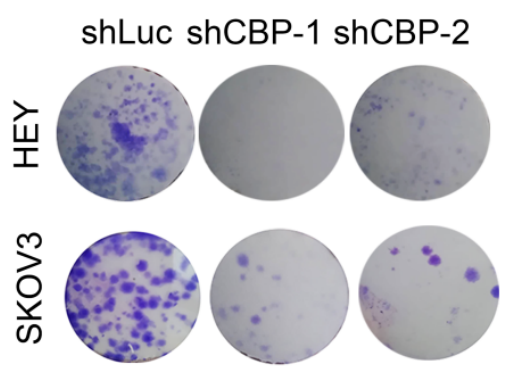

B

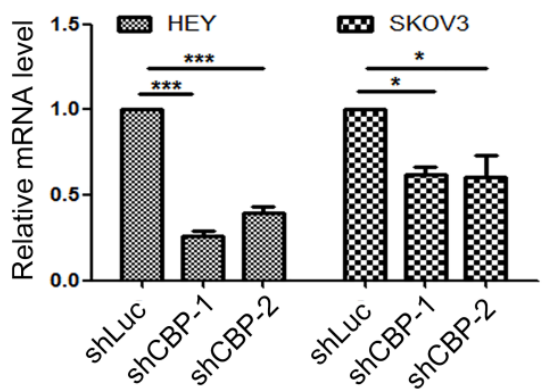

D

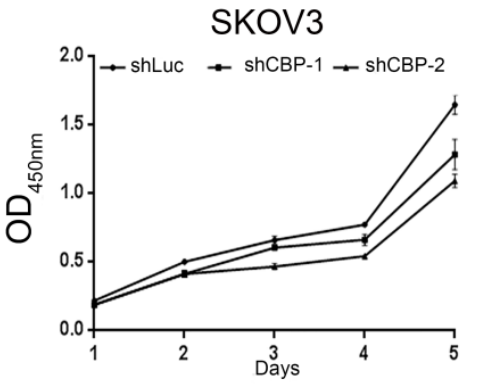

$\mathbf{F}$



Figure 2. CREBBP knockdown inhibits proliferation in ovarian cancer cells. A. Validation of CREBBP knockdown by western blot. B. Validation of CREBBP knockdown by GRT-PCR. C. CREBBP knockdwon inhibits proliferation in HEY cells. D. CREBBP knockdown inhibits proliferation in SKOV3 cells. E. CREBBP knockdown inhibits colony formation. F. Statistical analysis of colony numbers. 
apoptosis is crucial in the regulation of cellular proliferation and chemo-sensitivity, Annexin V-PI staining was performed to verify the effect of CREBBP knockdown on apoptosis in ovarian cancer cells. As the results shown, CREBBP knockdown enhanced CDDP-induced apoptosis in both HEY cells (Fig. 4E and $4 \mathrm{~F}$ ) and SKOV3 cells (Fig. 4G and 4H). Taken together, the results indicated that CREBBP knockdown promoted chemo-sensitivity, which was correlated with the enhanced apoptosis in ovarian cancer cells.

\section{CREBBP knockdown attenuates UPR}

To explore the underlying mechanism, we detected the expression of several proteins participating in unfolded protein response (UPR). As shown in Figure 5A, knockdown of CREBBP decreased GRP78 expression both in HEY and SKOV3 cells. We further detected proteins involving in the downstream signaling cascade: IRE1a (including IRE1 $a$ and XBP1), PERK (including PERK, p-eIF2a, ATF4 and STC2) and ATF6a. CREBBP knockdown reduced the expression of IRE1a (Fig. 5A), and the spliced XBP1 (sXBP1) mRNA was also reduced in CREBBP knockdown cells (Fig. 5B). Furthermore, expression of PERK, p-eIF2a, ATF4 and STC2 were dramatically decreased in CREBBP knockdown cells. However, no obvious change was found in the expression of ATF6a. Our results suggested that IRE1a and PERK signaling pathway were suppressed after CREBBP knockdown.

To verify whether UPR signaling pathway was involved in CREBBP knockdown-mediated apoptosis, we detected the expression of the aforementioned proteins in CDDP-treated ovarian cancer cells. As shown in Figure 5C, CDDP inhibited CBP expression and induced cleavage of Caspase 3. Moreover, Enhanced Caspase 3 cleavage occurred in CDDPtreated CREBBP knockdown cells. The result was in accordance with our previous apoptosis data (Fig. 4E), which indicated that CREBBP knockdown indeed promoted CDDP-induced apoptosis. We also found that both PERK, p-eIF2a, ATF4 and STC2 were down-regulated in CDDP-treated cells, and further decreased in CREBBP knockdown groups (Fig. 5C). Taken together, these results suggested that CREBBP knockdown attenuated UPR, and the PERK signaling pathway was involved in CREBBP knockdowninduced apoptosis.

\section{Discussion}

CBP is a crucial transcriptional co-activator in the regulation of gene expression via interacting with other transcription factors, such as SATB2, AP-1 and KLF3 [22-24]. CBP also acts as a lysine acetyltransferase to regulate protein expressions and functions via direct acetylation e.g., DOT1L, BCAT2, KDM2B and STX17 [14-16, 25]. Due to the transcription-promoting and LAT activity, CBP is involved in multiple cellular functions and
A

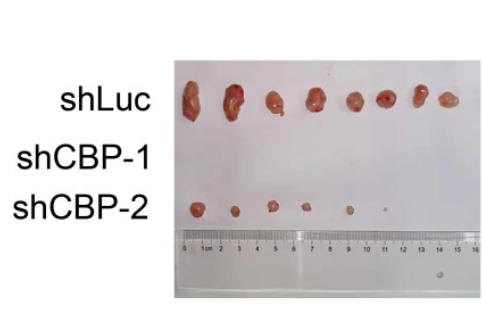

D

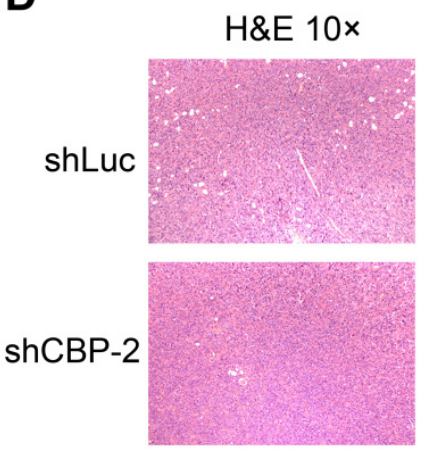

B



$H \& E 40 x$

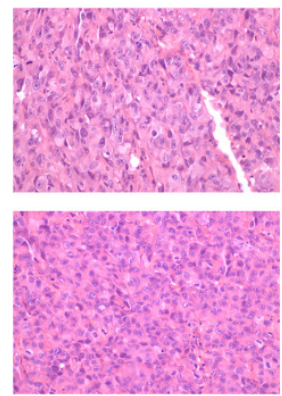

C
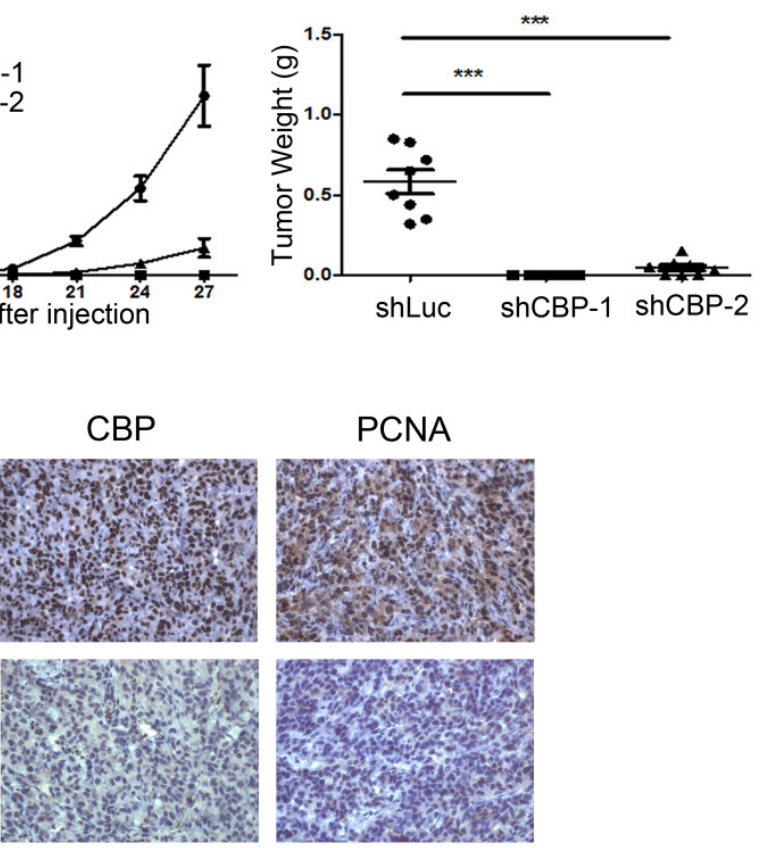

Figure 3. CREBBP knockdown inhibits tumorigenesis in xenograft tumor model. A. Representative images of tumors isolated from the nude mice. B. Summary of tumor volumes measured every three days. C. Tumor weights in nude mice at the $27^{\text {th }}$ day after inoculation. D. Images of hematoxylin and eosin staining and immunohistochemical staining for CBP and PCNA in xenograft tumors. 
pathological processes especially in tumorigenesis [26-30], but its effect on ovarian cancer has not been fully explored.

Our study showed that high expression of CBP predicted unfavorable prognosis in ovarian cancer patients. Knockdown of CREBBP by shRNA significantly suppressed tumor proliferation in ovarian cancer cells both in vitro and in vivo. Furthermore, CREBBP knockdown also enhanced the sensitivity of ovarian cancer cells to CDDP. IRE1a and PERK signaling pathway were suppressed by CREBBP knockdown, and the PERK signaling pathway participating in CREBBP knockdownmediated apoptosis.
A

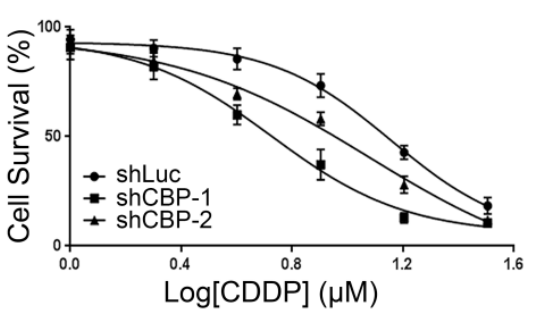

C

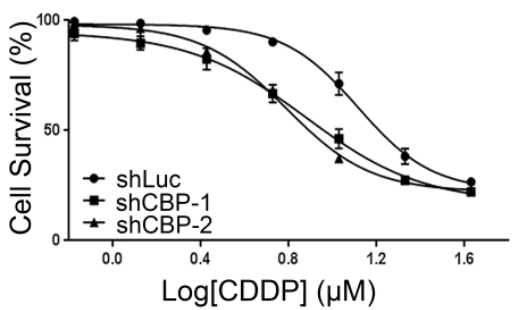

E

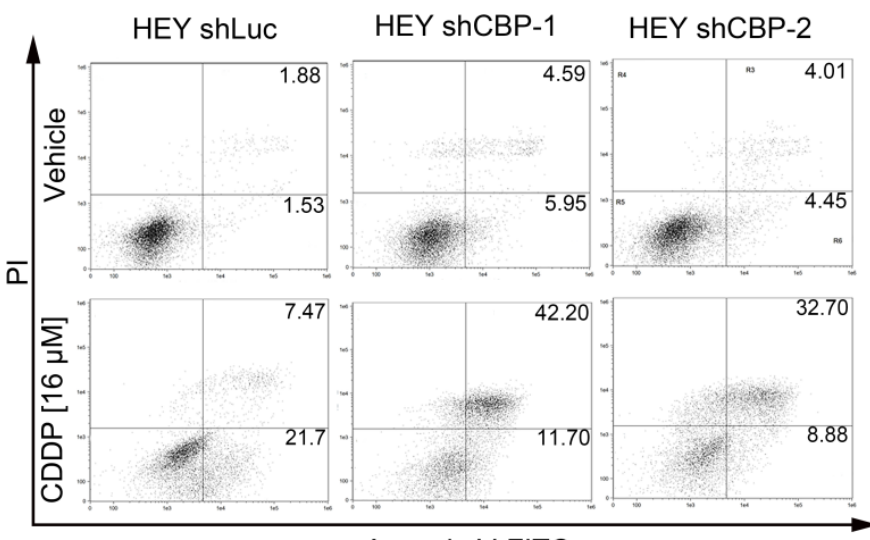

Annexin V-FITC

G

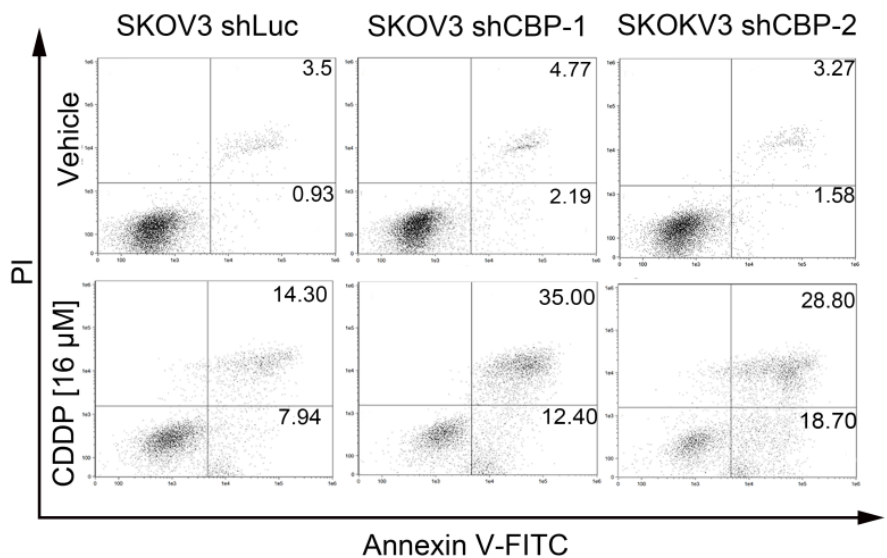

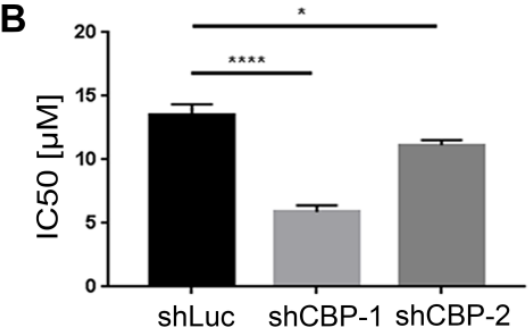

D

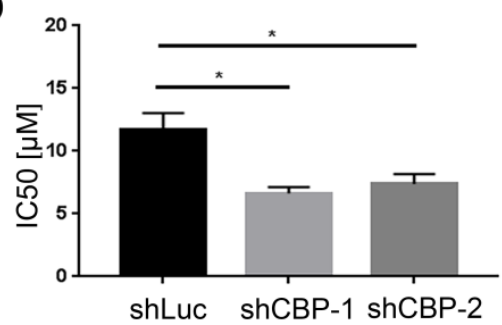

$\mathbf{F}$



H

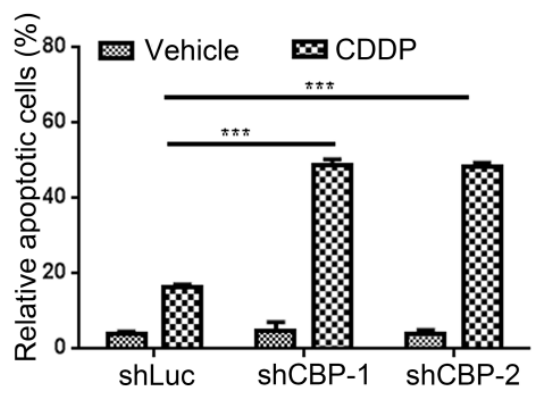

Figure 4. CREBBP knockdown promotes chemo-sensitivity and induces apoptosis in ovarian cancer cells. A. CREBBP knockdown promotes chemo-sensitivity in HEY cells. B. IC50 values of HEY cells. C. CREBBP knockdown promotes chemo-sensitivity in SKOV3 cells. D. IC50 values of SKOV3 cells. E. CREBBP knockdown enhances CDDP-induced apoptosis in HEY cells. F. Statistical analysis of apoptosis in HEY cells. G. CREBBP knockdown enhances CDDP-induced apoptosis in SKOV3 cells. H. Statistical analysis of apoptosis in SKOV3 cells. 


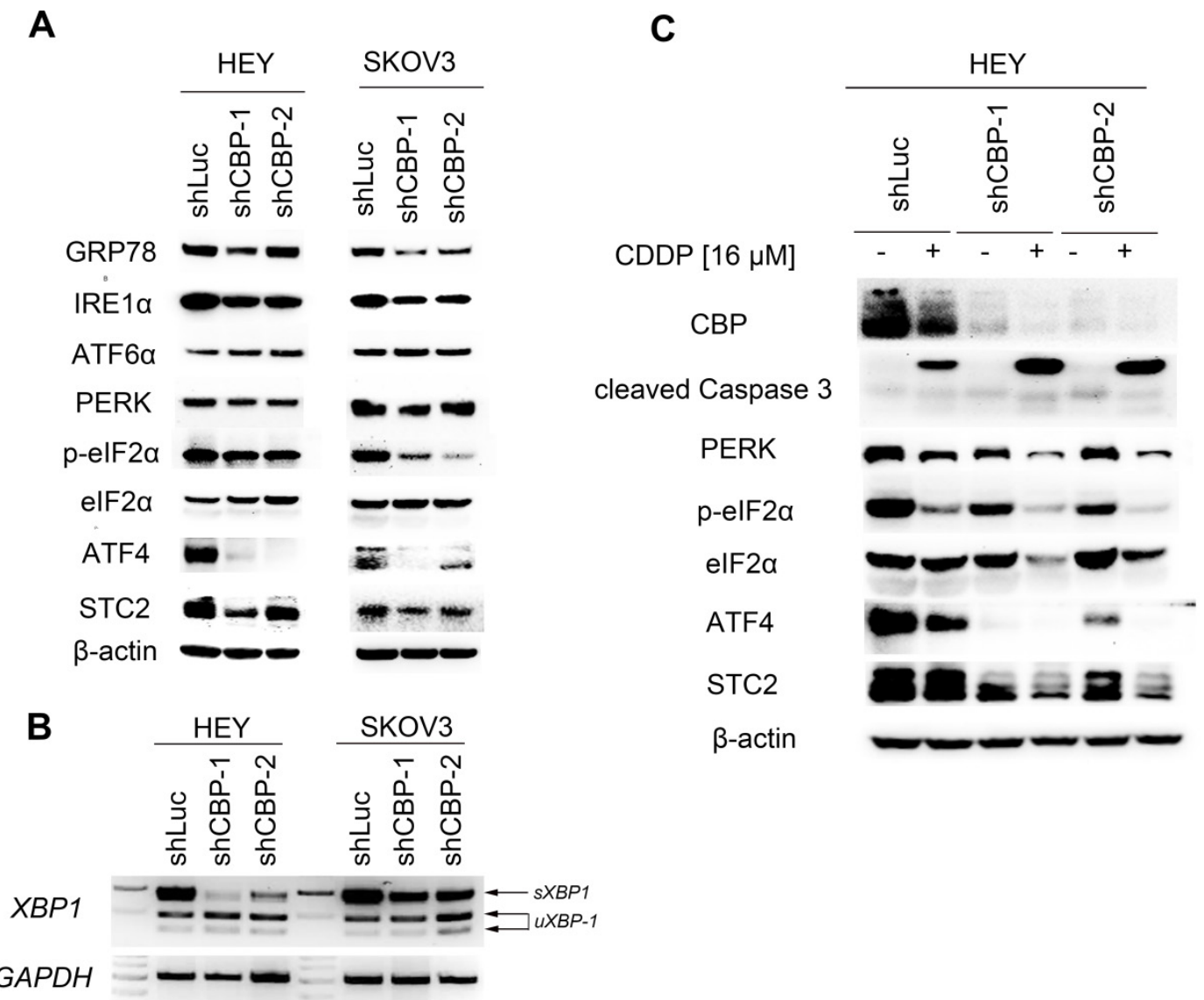

Figure 5. CREBBP knockdown attenuates UPR. A. CREBBP knockdown attenuates UPR examined by western blot. B. CREBBP knockdown reduces the level of $s X B P I$ examined by RT-PCR. C. PERK signaling pathway is involved in CBP-regulated proliferation and chemo-sensitivity.

Emerging evidence showed that activation of UPR signaling contributed to tumor development and was correlated with unfavorable prognosis in cancer patients [31, 32]. In EOC, GRP78, ATF6a and PERK were highly expressed in EOC tissues compared to the normal tissues, and correlated with advanced tumor stages. Moreover, high expression of GRP78 predicted poor overall survival (OS), suggesting that expression of UPR-associated proteins could be prognostic biomarkers in ovarian cancer [32]. Our study found that knockdown of CREBBP decreased GRP78 expression, suggesting low expression of CBP was correlated with good prognosis in ovarian cancer, which was in accordance with the results generated from the Kaplan-Meier Plotter database (Fig. 1).

PERK signaling pathway is the most rapidly activated among the three branches of UPR, followed by ATF6a and IRE1a sequentially [33]. STC2 has been reported to be regulated by PERK/ATF4 signaling pathway during UPR and exerts a cytoprotective effect [18]. In our study, we demonstrated that knockdown of CREBBP dramatically decreased PERK/ATF4/STC2 expression, and promoted apoptosis in ovarian cancer cells treated with CDDP. Previous studies showed that loss of PERK triggered oxidative DNA damage and ROS accumulation thus inhibiting tumorigenesis [34]. Therefore, further research is needed to explore whether oxidative stress was involved in CBP loss-induced tumor suppression.

Taken together, our results showed that high expression of CBP was correlated with poor prognosis in patients with ovarian cancer, and knockdown of CBP inhibited tumor growth, promoted chemosensitivity, and attenuated UPR in ovarian cancer cells. Our findings indicated that CBP could serve as a novel therapeutic target for ovarian cancer treatment.

\section{Acknowledgements}

The study was supported by National Natural Science Foundation of China (Grant No. 81602259 and 81702548).

\section{Competing Interests}

The authors have declared that no competing interest exists.

\section{References}

1. Siegel RL, Miller KD, Fuchs HE, Jemal A. Cancer Statistics, 2021. CA: a cancer journal for clinicians. 2021; 71: 7-33.

2. Sung H, Ferlay J, Siegel RL, Laversanne M, Soerjomataram I, Jemal A, et al. Global cancer statistics 2020: GLOBOCAN estimates of incidence and 
mortality worldwide for 36 cancers in 185 countries. CA: a cancer journal for clinicians. 2021; 71:209-49.

3. Lheureux S, Braunstein M, Oza AM. Epithelial ovarian cancer: Evolution of management in the era of precision medicine. CA: a cancer journal for clinicians. 2019; 69: 280-304.

4. Lheureux S, Gourley C, Vergote I, Oza AM. Epithelial ovarian cancer. Lancet. 2019; 393: 1240-53.

5. Yao WL, Wang TX, Huang FR. p300/CBP as a Key Nutritional Sensor for Hepatic Energy Homeostasis and Liver Fibrosis. Biomed Res Int. 2018;8168791.

6. Sharma N, Jadhav SP, Bapat SA. CREBBP re-arrangements affect protein function and lead to aberrant neuronal differentiation. Differentiation. 2010; 79: 218-31.

7. Zimmer SN, Zhou O, Zhou T, Cheng Z, Abboud-Werner SL, Horn D, et al. Crebbp haploinsufficiency in mice alters the bone marrow microenvironment, leading to loss of stem cells and excessive myelopoiesis. Blood. 2011; 118: 69-79.

8. Piccirillo AR, Cattley RT, D'Cruz LM, Hawse WF. Histone acetyltransferase CBP is critical for conventional effector and memory T-cell differentiation in mice. Journal of Biological Chemistry. 2019; 294: 2397-406.

9. Ward R, Johnson M, Shridhar V, van Deursen J, Couch FJ. CBP truncating mutations in ovarian cancer. Journal of medical genetics. 2005; 42: 514-8.

10. Mullighan CG, Zhang J, Kasper LH, Lerach S, Payne-Turner D, Phillips LA, et al. CREBBP mutations in relapsed acute lymphoblastic leukaemia. Nature. 2011; 471: 235-9.

11. Camos M, Esteve J, Jares P, Colomer D, Rozman M, Villamor N, et al. Gene expression profiling of acute myeloid leukemia with translocation $\mathrm{t}(8 ; 16)(\mathrm{p} 11 ; \mathrm{p} 13)$ and MYST3-CREBBP rearrangement reveals a distinctive signature with a specific pattern of HOX gene expression. Cancer Res. 2006; 66: 6947-54

12. Ding LY, Chen SA, Liu P, Pan YQ, Zhong J, Regan KM, et al. CBP Loss Cooperates with PTEN Haploinsufficiency to Drive Prostate Cancer: Implications for Epigenetic Therapy. Cancer Res. 2014; 74: 2050-61.

13. Jia D, Augert A, Kim DW, Eastwood E, Wu N, Ibrahim AH, et al. Crebbp Loss Drives Small Cell Lung Cancer and Increases Sensitivity to HDAC Inhibition. Cancer discovery. 2018; 8: 1422-37.

14. Lei MZ, Li XX, Zhang Y, Li JT, Zhang F, Wang YP, et al. Acetylation promotes BCAT2 degradation to suppress BCAA catabolism and pancreatic cancer growth. Signal transduction and targeted therapy. 2020; 5: 70.

15. Liu C, Yang Q, Zhu Q, Lu X, Li M, Hou T, et al. CBP mediated DOT1L acetylation confers DOT1L stability and promotes cancer metastasis. Theranostics. 2020; 10: 1758-76.

16. Qi Y, Zhao Y. CBP-triggered KDM2B acetylation accelerates the carcinogenesis of colon cancer. J Cell Physiol. 2020; 235: 2901-10.

17. Madden E, Logue SE, Healy SJ, Manie S, Samali A. The role of the unfolded protein response in cancer progression: From oncogenesis to chemoresistance. Biology of the cell. 2019; 111: 1-17.

18. Ito D, Walker JR, Thompson CS, Moroz I, Lin W, Veselits ML, et al. Characterization of stanniocalcin 2, a novel target of the mammalian unfolded protein response with cytoprotective properties. Mol Cell Biol. 2004; 24: 9456-69.

19. Tsuru A, Imai $Y$, Saito $M$, Kohno $K$. Novel mechanism of enhancing IRE1alpha-XBP1 signalling via the PERK-ATF4 pathway. Sci Rep. 2016; 6: 24217.

20. Yoshida H, Matsui T, Yamamoto A, Okada T, Mori K. XBP1 mRNA is induced by ATF6 and spliced by IRE1 in response to ER stress to produce a highly active transcription factor. Cell. 2001; 107: 881-91.

21. Teske BF, Wek SA, Bunpo P, Cundiff JK, McClintick JN, Anthony TG, et al. The eIF2 kinase PERK and the integrated stress response facilitate activation of ATF6 during endoplasmic reticulum stress. Mol Biol Cell. 2011; 22: 4390-405.

22. Tao $W$, Zhang A, Zhai $K$, Huang $Z$, Huang $H$, Zhou $W$, et al. SATB2 drives glioblastoma growth by recruiting CBP to promote FOXM1 expression in glioma stem cells. Embo Mol Med. 2020; 12: e12291.

23. Bejjani F, Tolza C, Boulanger M, Downes D, Romero R, Maqbool MA, et al. Fra-1 regulates its target genes via binding to remote enhancers without exerting major control on chromatin architecture in triple negative breast cancers. Nucleic Acids Res. 2021;49:2488-508.

24. Jones J, Chen YF, Tiwari M, Li JT, Ling J, Sen GL. KLF3 Mediates Epidermal Differentiation through the Epigenomic Writer CBP. iScience. 2020; 23: 101320.

25. Shen QH, Shi Y, Liu JQ, Su H, Huang JT, Zhang Y, et al. Acetylation of STX17 (syntaxin 17) controls autophagosome maturation. Autophagy. 2020; 17:1157-69.

26. Dutto I, Scalera C, Prosperi E. CREBBP and p300 lysine acetyl transferases in the DNA damage response. Cell Mol Life Sci. 2018; 75: 1325-38.

27. Schoof M, Launspach M, Holdhof D, Nguyen L, Engel V, Filser S, et al. The transcriptional coactivator and histone acetyltransferase CBP regulates neural precursor cell development and migration. Acta Neuropathol Com. 2019; 7: 199.

28. Nagata DED, Chiang EY, Jhunjhunwala S, Caplazi P, Arumugam V, Modrusan Z, et al. Regulation of Tumor-Associated Myeloid Cell Activity by CBP/EP300 Bromodomain Modulation of H3K27 Acetylation. Cell Rep. 2019; 27: $269-81$

29. Castillo J, Wu E, Lowe C, Srinivasan S, McCord R, Wagle MC, et al. CBP/p300 Drives the Differentiation of Regulatory T Cells through Transcriptional and Non-Transcriptional Mechanisms. Cancer Res. 2019; 79: 3916-27.
30. Attar N, Kurdistani SK. Exploitation of EP300 and CREBBP Lysine Acetyltransferases by Cancer. Cold Spring Harbor perspectives in medicine. 2017; 7: a026534

31. Liu JN, Xiao ML, Li JJ, Wang DL, He YF, He J, et al. Activation of UPR Signaling Pathway is Associated With the Malignant Progression and Poor Prognosis in Prostate Cancer. Prostate. 2017; 77: 274-81.

32. Samanta S, Tamura S, Dubeau L, Mhawech-Fauceglia P, Miyagi Y, Kato H, et al. Clinicopathological significance of endoplasmic reticulum stress proteins in ovarian carcinoma. Sci Rep. 2020; 10: 2160.

33. Siwecka N, Rozpedek W, Pytel D, Wawrzynkiewicz A, Dziki A, Dziki L, et al. Dual role of Endoplasmic Reticulum Stress-Mediated Unfolded Protein Response Signaling Pathway in Carcinogenesis. International journal of molecular sciences. 2019; 20: 4354

34. Bobrovnikova-Marjon E, Grigoriadou C, Pytel D, Zhang F, Ye J, Koumenis C, et al. PERK promotes cancer cell proliferation and tumor growth by limiting oxidative DNA damage. Oncogene. 2010; 29: 3881-95. 